\title{
Multi probes measurements at the PALS Facility Research Centre during high intense laser pulse interactions with various target materials
}

\author{
Massimo De Marco ${ }^{1,2, *}$, Josef Krása ${ }^{1}$, Jakub Cikhardt ${ }^{3,5}$, Fabrizio Consoli ${ }^{4}$, Riccardo De Angelis ${ }^{4}$, Miroslav Pfeifer $^{1,3}$, \\ Miroslav Krůs ${ }^{1,3}$, Jan Dostál ${ }^{1,3}$, Daniele Margarone ${ }^{1}$, Antonino Picciotto ${ }^{6}$, Andriy Velyhan ${ }^{1}$, Daniel Klír ${ }^{3,5}$, Roman \\ Dudžák ${ }^{1,3}$, Jiři Limpouch ${ }^{2}$ and Georg Korn ${ }^{1}$ \\ ${ }^{1}$ Institute of Physics CAS, v. v. i., Na Slovance 2, 18221 Prague, Czech Republic \\ ${ }^{2}$ Czech Technical University in Prague, FNSPE, Břehavá 7, 11519 Prague 1, Czech Republic \\ ${ }^{3}$ Institute of Plasma Physics CAS,Za Slovankou 3, 18200 Prague 8, Czech Republic \\ ${ }^{4}$ ENEA, Fusion and Nuclear Safety Department, C.R. Frascati, Via E. Fermi 45, 00044 Frascati, Italy \\ ${ }^{5}$ Czech Technical University in Prague, Faculty of Electrical Engineering, Technická 2, 16627 Prague 6, Czech Republic \\ ${ }^{6}$ Micro-Nano Facility, Fondazione Bruno Kessler, 38123 Trento, Italy
}

\begin{abstract}
During the interaction of high intense laser pulse with solid target, a large amount of hot electrons is produced and a giant Electromagnetic Pulse (EMP) is generated due to the current flowing into the system target-target holder, as well as due to the escaping charged particles in vacuum. EMP production for different target materials is investigated inside and outside the target chamber, using monopole antenna, super wide-band microstrip antenna and Moebius antenna. The EMP consists in a fast transient magnetic field lasting hundreds of nanosecond with frequencies ranging from $\mathrm{MHz}$ to tens of GHz. Measurements of magnetic field and return target current in the range of kA were carried out by an inductive target probe (Cikhardt J. et al. Rev. Sci. Instrum. 85 (2014) 103507).
\end{abstract}

\section{Introduction}

When high intense laser pulse interacts with a solid target a strong electromagnetic pulse (EMP) is generated. The main sources of EMP are the return target current flowing through the target holder and the charged particles escaping into the vacuum; both these EMP sources are directly connected with hot electrons escaping the plasma [1-8]. To obtain a complex data set on this process, various probes are employed for observation of return current and EMP emission during 3TW PALS laser pulse-target interaction experiment. While the target current probe (T-Probe) allowed us to determine occurred current onto the target as well as magnetic field around the target holder, loop antennas and linear antennas were employed for measurements of electric field and magnetic field both inside and outside the interaction chamber.

The escaping electrons generate up to hundreds $\mathrm{kV}$ potential at the target and 10kA current flows from the target to the ground [9-12]. The return current, that compensates the net positive target charge, generates an electromagnetic pulse because the system target-target holder is in first approximation a linear antenna emitting toroidal radiation pattern in the far-field and more complex field pattern in the near field zone [13-20]. The charged particles moving inside the target chamber produce the Lenard-Wiechert potentials with radiative and nonradiative electric and magnetic fields depending on their both velocity and acceleration [21-24].

The generated EMP is a transient pulse lasting hundreds nanoseconds which is much longer than

*Corresponding author: massimo.demarco@eli-beams.eu 
laser pulse target interaction [25-28]. The frequencies range from $\mathrm{MHz}$ to tens $\mathrm{GHz}$.

EMP propagates in all directions and it resonates at the resonant frequencies of the target chamber that acts as not efficient resonant cavity with many leakages (e.g. big glass windows) [29]. The presence of the EMP inside the vacuum chamber as well as in the experimental hall can cause problems with sensitive diagnostics, sophisticated data acquisition systems and, in some case, even disruptions of electronic devices can occur.

The generation of this harmful EMP depends on laser pulse characteristics, target material and shape of the target chamber as well as accessories located within it.

This contribution reports measurement carried out at PALS facility and it is focused on characterizing the magnetic field using different antennas.

\section{Method and results}

The employed target current probe [20] makes it possible to measure magnetic field at $5 \mathrm{~mm}$ distance from the target holder, which is generated only by the neutralizing target current. Fig. 1 shows the increase of the magnetic field associated with target-holder current with the enhancement of the laser energy.

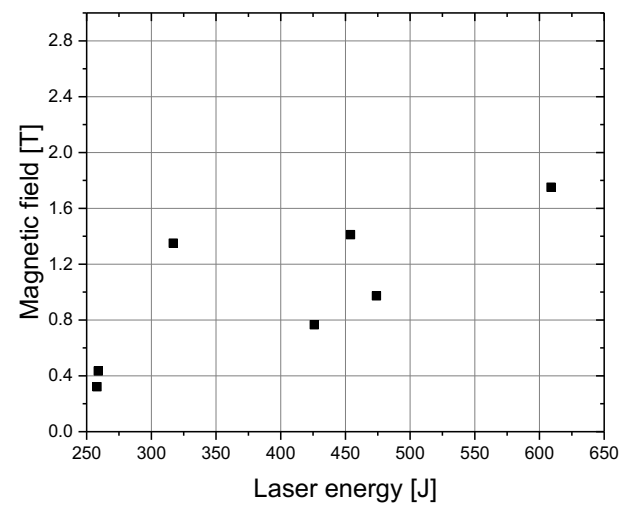

Fig.1 Laser pulse energy dependence of magnetic field produced by return target current which was detected by the inductive target probe at $5 \mathrm{~mm}$ distance from the target holder. Target material was Tantalum.

The measurements of magnetic field generated by only the return current for different target materials, were carried out by using the target current probe [20] and they are presented in Fig.2(a). The magnetic field was normalized to the laser energy $E_{L}$, see Fig.2(b). The target material influence on the return target current is also affected by the amount of impurities chemisorbed on the irradiated target surfaces which significantly contribute to the emission of hot electrons [18]. For this reason, the study of the relation between target material and hot electron emission should be performed with perfectly cleaned targets and under ultra-high vacuum conditions.

(a)

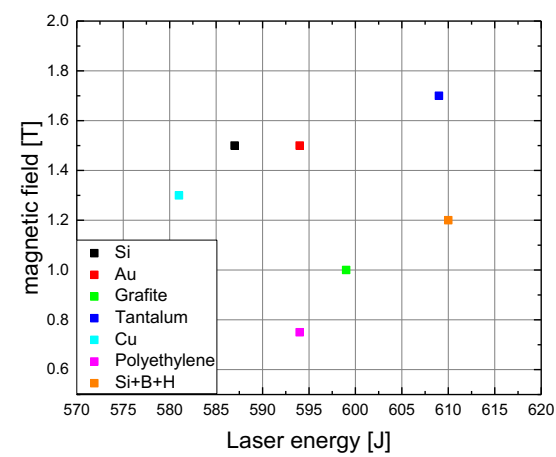

(b)

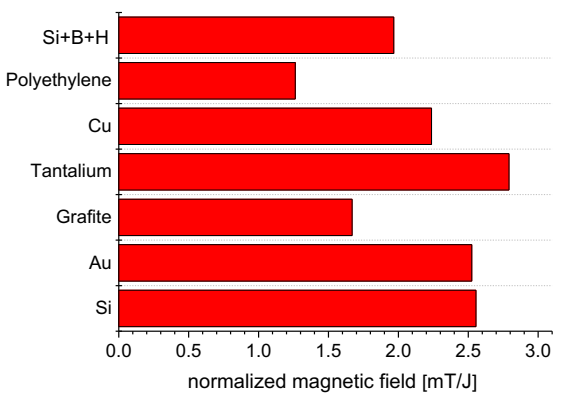

Fig.2 (a) Magnetic field at target holder, measured by the T-Probe, for different target materials. (b) Normalized magnetic field with $\mathrm{E}_{\mathrm{L}}$.

Moebius antennas were used outside the interaction chamber and placed at $3 \mathrm{~m}$ distance from the PALS spherical chamber wall. The highest EMP signal was detected when the Moebius antenna was placed in front of the glass window $(40 \mathrm{~cm}$ in diameter) serving as an input window into the chamber for the laser beam. The typical EMP signals and frequency spectra were collected for different target materials. The spectra have two dominant peaks, one between $500 \mathrm{MHz}$ and $1 \mathrm{GHz}$ and another between $1 \mathrm{GHz}$ and $1.5 \mathrm{GHz}$. Although at the moment it is not clear the correlation of the EMP spectra with the target materials, one can presume the influence of the target surface impurities as hydrocarbons on the emission of hot electrons and consequently on the EMP intensity, as Fig. 3 shows. The tantalum target exhibits the highest amplitude of the first peak (see Fig. 3a) probably denoting big amount of hydrocarbon impurities on its surface [9]. This clearly exceeds the EMP amplitudes registered for polyethylene and graphite targets. 
(a)
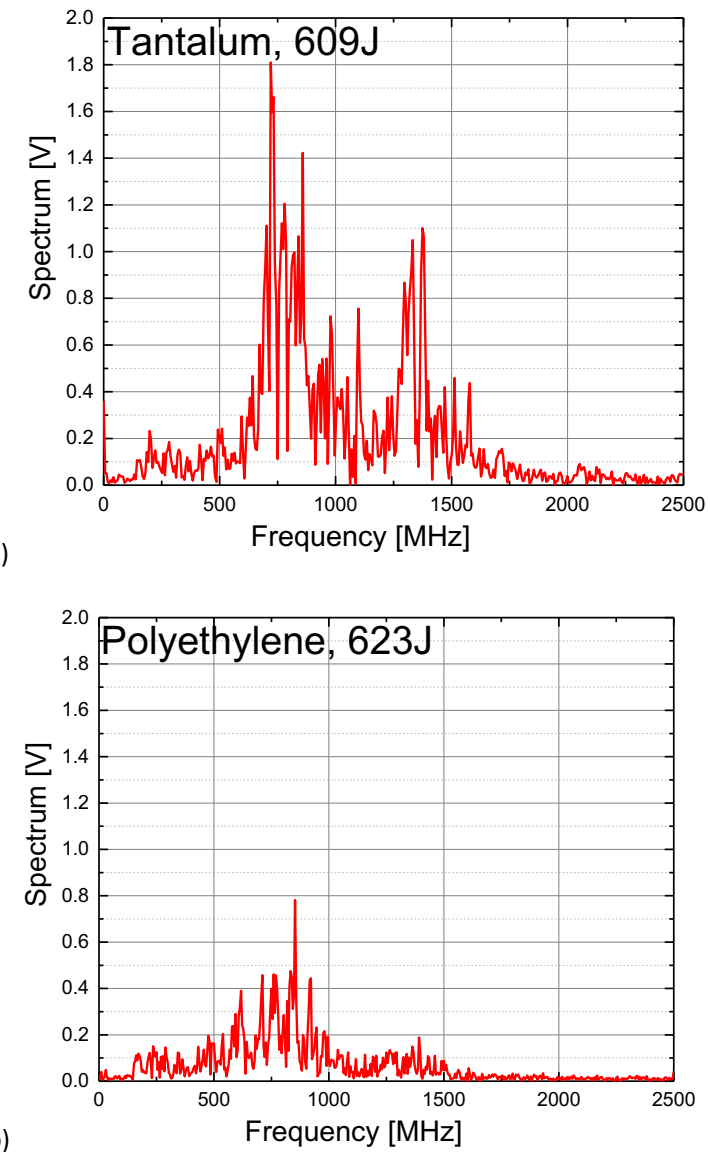

(b)

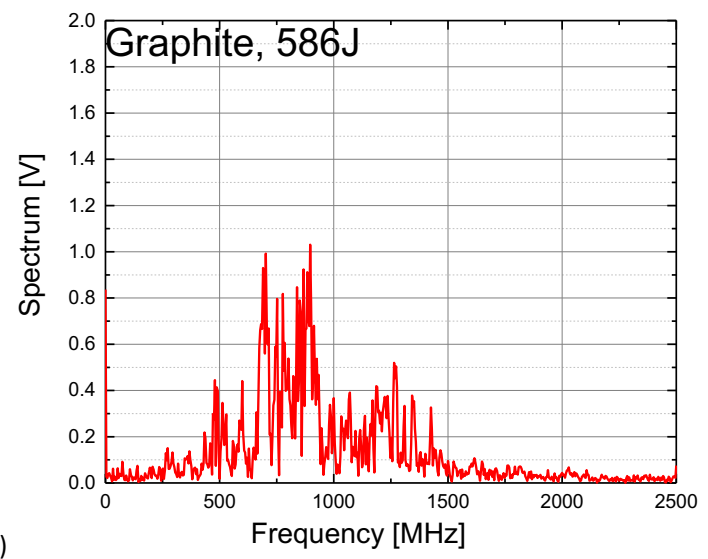

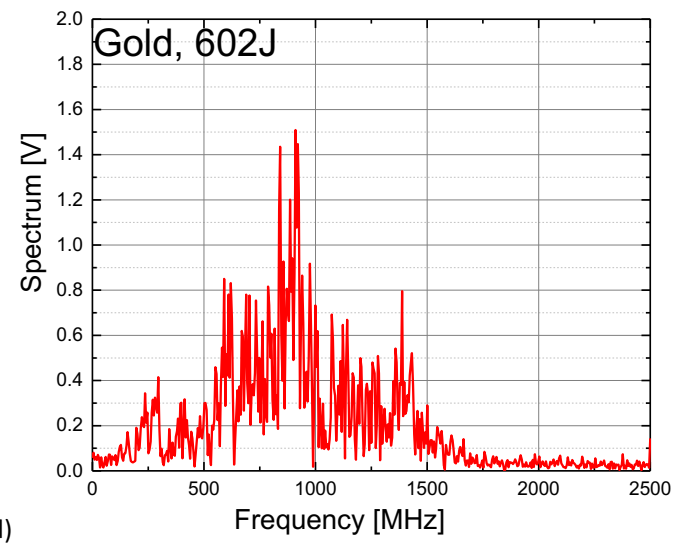

Fig.3 Frequency spectra of EMP emitted by interaction of laser pulse with various target under similar conditions (laser energy $586-623 \mathrm{~J}$ ) detected by Moebius antenna outside the interaction chamber at $3 \mathrm{~m}$ distance from the glass window. (a) Tantalum, (b) Polyethylene, (c) Graphite and (d) Gold.

Double exponential fits [14] were carried out for each detected EMP signal and both the time duration and rise time duration of the magnetic flux were determined; the mean values of rise time and FWHM time duration over 10 shots are collected in Fig.4.

The rise time is a parameter that reflects the rate of charge particles emission from target and it can be directly connected with both the atomic structure of the target material (e.g. atomic number) and the absorption of impurities on the target surface. In the case of Graphite target, the magnetic flux rise time reaches a very low value of only $8 \mathrm{~ns}$ while for the case of Tantalum and $\mathrm{Si}+\mathrm{B}+\mathrm{H}[30]$ the highest values are reached. In the case of Copper, Silicon, Gold and Polyethylene the rise time is very similar.

The time duration of the FWHM has a minimum for Gold at $81 \mathrm{~ns}$ and a maximum for Tantalum, Polyethylene and $\mathrm{Si}+\mathrm{B}+\mathrm{H}[30]$ at about $110 \mathrm{~ns}$. The FWHM time durations of magnetic flux for different target materials are very similar and it is probably due to the same geometry of the used target chamber that plays an important role for the determination of the signals decays [19]. 
(a)

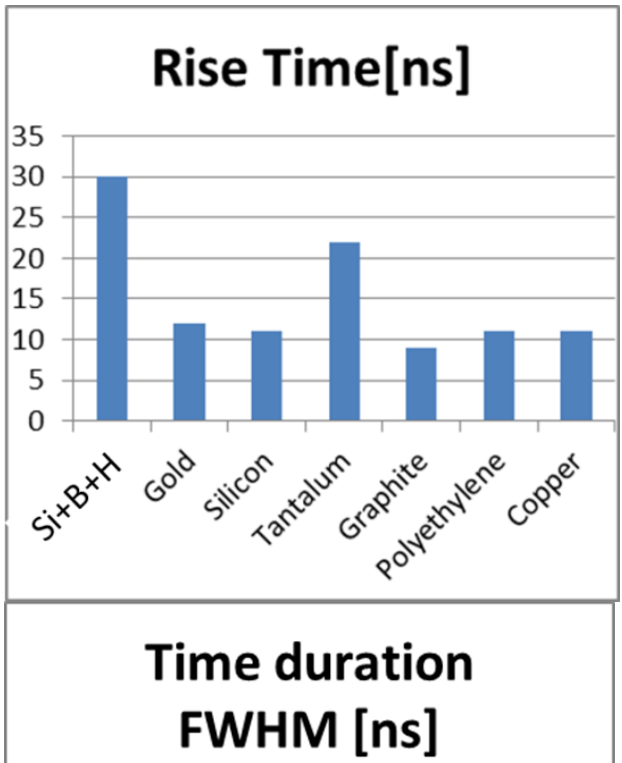

(b)

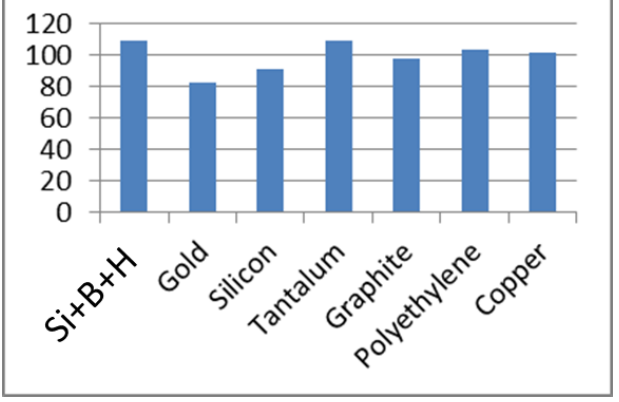

Fig.4 Mean rise time (a) and mean FWHM time duration (b) of EMP for different target materials.

The signal acquisitions carried out by linear monopole antenna [31] and super wide-band antenna [32] placed both inside the interaction chamber, above the target at $30 \mathrm{~cm}$ from it, and protected by a thick stainless-steel slab, as from Fig.5(c), are shown in Fig.5(a) and in Fig.5(b), respectively. It is well known that it is very difficult to get reliable measurements of EMP by conductive probes when high intensity and energetic lasers are used [1-10, 12]. In fact, big fluctuations were present shot by shot on the measurements, due to background noise on the oscilloscopes caused by bad EMC-shielding of the scope and by spurious coupling of EMP with outside conductor of the coaxial cables. To resolve these problems, it was necessary to use a delay line to clean the signals, as described in references [33, 34]. Fig. 5 shows the laser energy dependence of the EMP amplitude for various target and antennas. For energies higher than $400 \mathrm{~J}$, high values of EMP were obtained for gold target, and this is in good agreement with results from Fig. 2a achieved by Tprobe. Unfortunately, we were not able to get reliable results from these probes for all the other materials studied, and for example for Tantalum. A clear and more complete comparison of absorbed EMP for other target materials, will be thus performed in future experimental campaigns [34].
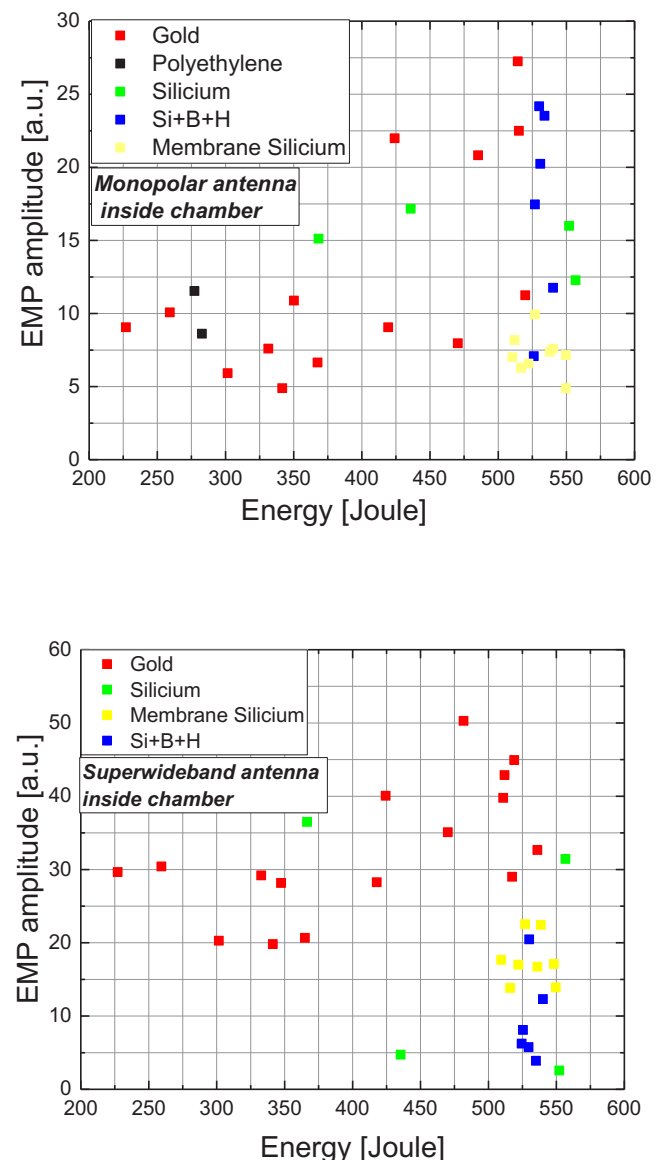

(b)

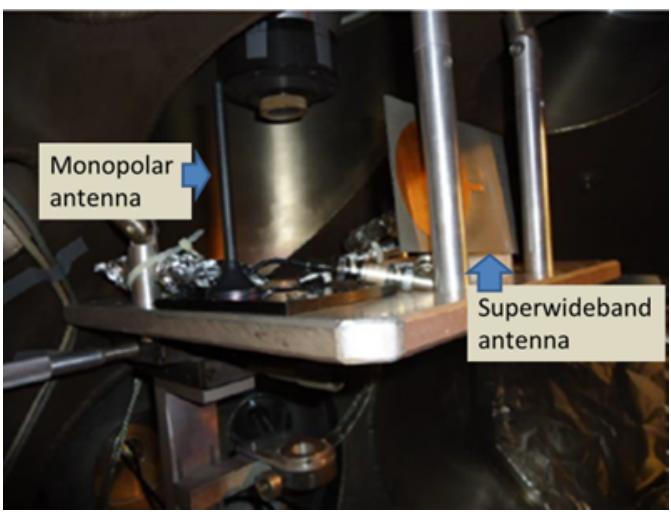

Fig.5 EMP amplitude for monopolar antenna (a) and SWB antenna (b) inside the experimental chamber; picture of the SWB magnetic antenna and linear antenna placed inside the target chamber at $30 \mathrm{~cm}$ above the laser target interaction point (c). 


\section{Conclusions}

In this work the results of EMP measurements are shown for different probes. The T-Probe allows us to measure the magnetic field produced only with the return target current. It was shown how the magnetic field increases with the laser pulse energy, as the target current scales with the laser intensity as $\sqrt[3]{I \lambda^{2}}$ [20]. The analysis of the collected data for various materials, show that EMP spectra have two dominant peaks which change in their ratios based on the target material; however their dependence is not yet understood. Nevertheless the influence of hydrogen or carbon impurities on the emission of hot electrons and, thus, on the EMP intensity seems to be evident. The EMP rise time and duration (FWHM) was determined for each of the calculated magnetic flux. Average over 10 shots shows that Graphite presents the lowest rise time while Tantalum and $\mathrm{Si}+\mathrm{B}+\mathrm{H}$ targets shows the highest ones. For different target materials, the time duration at FWHM is very similar and it is related to the common target chamber structure that induces similar decaying of the EMP pulses. Measurements performed by conductive antennas inside the chamber suffered of high background noise, but it was possible to find a suitable solution and reliable results were eventually achieved, which are in rough agreement with those from T-probe. More complete characterization and comparison of results for different target materials can be performed in future experimental campaigns.

The PALS staff and other researchers participating in the experimental campaign greatly appreciate the financial support from the Czech Ministry of Education within the project "Creating and probing dense plasma at the PALS facility" (CZ.02.1.01/0.0/0.0/16_013/0001552), and European Regional Development - the project ELI: Extreme Light Infrastructure (CZ.02.1.01/0.0/0.0/15_008/0000162). The research leading to these results has also received funding from the Czech Science Foundation (Grant No. 16-07036S). The support from the projects no. CZ.1.07/2.3.00/20.0087 and CZ.1.07/2.3.00/20.0279, which were co-financed by the European Social Fund and the state budget of the Czech Republic are also acknowledged. The work of F.C and R.D.A. has been partially carried out within the framework of the EUROfusion Consortium and has received funding from the Euratom research and training programme 20142018 under grant agreement No. 633053. The views and opinions expressed herein do not necessarily reflect those of the European Commission. The support by the Ministry of Education, Youth and Sports of the Czech Republic, Project No.: LM2015083 (PALS RI), is also gratefully acknowledged.

\section{References}

1. M. J. Mead et al., Rev. Sci. Instrum. 75, 4225 (2004).

2. M. De Marco et al., J. Phys.: Conf. Ser. 508 (2014) 012007.

3. M. De Marco et al., Physics of Plasmas 24, 083103 (2017)

4. J.-L.Dubois et al., Phys. Rev. E 89, 013102 (2014).

5. R.F. Benjamin et al., Phys. Rev. Lett. 42 (1979) 890-893.

6. F. Consoli et al., Scientific Reports 6, 27889 (2016).

7. C. G. Brown et al., Rev. Sci. Instrum. 83, 10D729 (2012).

8. C G Brown Jr. et al., J. Phys.: Conf. Series 112, 032025 (2008).

9. J. Krása et al., Laser Part. Beams 33, 601-605 (2015).

10. J.-L.Dubois et al., Phys. Rev. E 89, 013102 (2014).

11. S. Ramo et al., Fields and Waves in Communication Electronics (Wiley, New York, 1965), p.541.

12. A. Poyé et al.. Phys. Rev. E 91, 043106 (2015).

13. M. G. Drouet and H. Pépin. Appl. Phys. Lett. 28, 426-428 (1976).

14. Krása, et al., Plasma Phys. Control. Fusion 59 065007 (2017).

15. F. Consoli et al., Environment and Electrical Engineering (EEEIC), 2015 IEEE 15th International Conference on, Rome, 2015, pp. 182-187.

16. M. G. Drouet and R. Bolton. Phys. Rev. Lett. 36, 591-594 (1976).

17. C. J. Price et al., Rev. Sci. Instrum. 86, 033502 (2015).

18. A. Poyé et al., Phys. Rev. E 92, 043107 (2015).

19. J. Krása et al., Laser Part. Beams 32, 15-20 (2014).

20. M. De Marco et al., JINST 11, C06004 (2016).

21. J. Cikhardt et al., Rev. Sci. Instrum. 85, 103507 (2014).

22. C.G. Brown et al. Rev. Sci. Instrum. 83 (2012) 10D729.

23. C.G. Brown Jr. et al., J. Phys.: Conf. Ser. 244 (2010) 032001

24. Zi-Yu Chen et al., Phys. Scr. 83, 055503 (2011). 
25. V. Nassisi and A. Luches, Rev. Sci. Instrum. 50 (1979) 900-902.

26. Hyoung Suk Kim. Electromagnetic Waves in Cavity Desig. Ed.: Ali Akdagli, ISBN: 978-953307-302-6, InTech 2011, p. 77-100.

27. M. De Marco et al., Nukleonika (2015) 10.1515.

28. F. S. Felber. Appl. Phys. Lett. 86, 231501 (2005)

29. F. Consoli et al Physics Procedia 62 ( 2015) $11-$ 17

30. A. Picciotto et al, Phys. Rev. X, 4, 031030 (2014

31. SPE-12-8-046/E/SS, Technical Report, Taoglas, 2009

32. S. Barbarino, F. Consoli, IEEE Trans. Antennas Propag. 58 (2010) 4074

33. M. De Marco, et al, Journal of Physics: Conference Series 508 (2014) 012007

34. F. Consoli et al, submitted to the European Physical Journal Web Conferences 